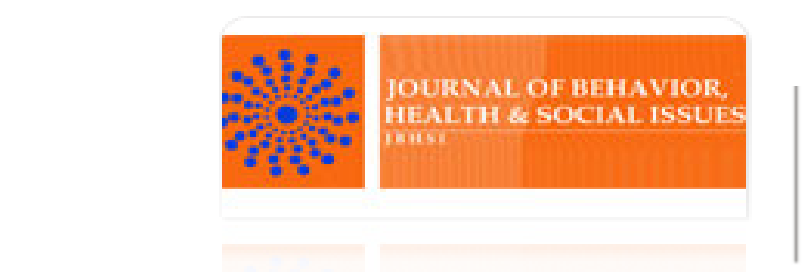

Journal of Behavior, Health \& Social

Issues

ISSN: 2007-0780

jcpedro@unam.mx

Asociación Mexicana de Comportamiento y Salud, A. C.

México

Pulido-Avalos, Lizbeth; Rangel-Bernal, Nora; Ortiz-Rueda, Gerardo EFECTO DEL TIPO DE CONTINGENCIA EN EL ESTABLECIMIENTO Y CUALIDAD DE INTERCAMBIOS VERBALES: SU PAPEL EN LA ELECCIÓN DE ALTERNATIVAS COMPARTIDAS Journal of Behavior, Health \& Social Issues, vol. 6, núm. 2, noviembre, 2014, pp. 71-86 Asociación Mexicana de Comportamiento y Salud, A. C.

Distrito Federal, México

Disponible en: http://www.redalyc.org/articulo.oa?id=282235731006

Cómo citar el artículo

- Número completo

- Más información del artículo

- Página de la revista en redalyc.org

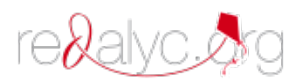

Sistema de Información Científica Red de Revistas Científicas de América Latina, el Caribe, España y Portugal Proyecto académico sin fines de lucro, desarrollado bajo la iniciativa de acceso abierto 


\title{
EFECTO DEL TIPO DE CONTINGENCIA EN EL ESTABLECIMIENTO Y CUALIDAD DE INTERCAMBIOS VERBALES: SU PAPEL EN LA ELECCIÓN DE ALTERNATIVAS COMPARTIDAS
}

\author{
EFFECT OF THE CONTINGENCY TYPE ON THE VERBAL EXCHANGES \\ ESTABLISHMENT AND QUALITY: THEIR ROLE ON SHARED \\ ALTERNATIVES CHOICES
}

\author{
Lizbeth Pulido-Avalos \\ Universidad Veracruzana. Veracruz, México. \\ Nora Rangel-Bernal \\ Universidad de Guadalajara, Guadalajara, México \\ Gerardo Ortiz-Rueda \\ Universidad de Guadalajara, Guadalajara, México
}

\author{
Recibido: Enero 3, 2014 \\ Revisado: Octubre 6, 2014 \\ Aceptado: Octubre 28, 2014
}

El presente estudio evaluó el papel de la exposición ante diferentes tipos de arreglos contingenciales en el establecimiento y la cualidad de los intercambios verbales que los participantes establecieron así como su efecto en la elección de alternativas compartidas de altruismo parcial.

Para ello, se empleó una tarea de armado de rompecabezas virtuales, mismos que fueron presentados en dos computadoras que podían estar o no interconectadas en red dependiendo de la condición experimental en turno, de acuerdo con la propuesta teórico-metodológica de Ribes (2001) para el estudio de la conducta social desde una perspectiva psicológica. Los participantes fueron colocados en una misma sala o en salas experimentales diferentes, a fin de manipular la posibilidad o imposibilidad de que establecieran intercambios verbales de cualquier tipo y, de esta manera, sopesar su efecto en la elección de alternativas compartidas de altruismo parcial.

En general, se encontró que el tipo de contingencia a la que los participantes fueron expuestos se relacionó de manera directa con la cualidad de los intercambios verbales que estos mismos establecieron, y que la elección de alternativas compartidas de altruismo parcial se vio favorecida por la presencia de intercambios verbales de cualidad predominantemente estratégica.

Palabras clave: Contingencia no compartida; contingencia compartida; intercambio verbal; altruismo parcial; niños de primaria. 


\section{Abstract}

This study evaluated the role of the exposure to different kinds of contingential arrays (non-shared individual, non-shared collective and shared) within the establishment and quality (in terms of its particular, general or strategic character) of the verbal exchanges that the experimental participants established, as well as its effect on the election of shared alternatives of partial altruism.

A virtual puzzle solving task was used, puzzles were displayed in two computers that could be or not connected on a network, depending on the experimental condition in turn. The experimental sequences were planned on a way where their fundamental difference resided on the exposure time to non-shared contingencies before the choice conditions between shared or non-shared contingencies of partial altruism, based on Ribes (2001) theorical-methodological framework for social behavior study from a psychological perspective. Participants were placed in the same or in different experimental rooms in order to achieve maximum control over their possibility or not to establish verbal exchanges of any kind and to be able to assess its effect on the choice of shared alternatives of partial altruism.

In general, it was found that the kind of contingency to which participants were exposed was directly related to the quality of the verbal exchanges that they established between them, and that the choice of shared alternatives of partial altruism was favored by the presence of verbal exchanges of strategic quality.

Key words: Not shared contingency; shared contingency; verbal exchange; partial altruism; elementary school children students.

\section{Introducción}

El estudio de la llamada conducta cooperativa, considerada como un caso central y representativo de la conducta social (Schmitt, 1998), ha sido emprendido desde diversas perspectivas. El Paradigma Operante del Análisis Experimental de la Conducta y la Teoría de Juegos son ejemplos de perspectivas cuyos enunciados fundamentales para explicar la conducta cooperativa descansan en un principio económico-utilitario básico: el de las ganancias y su posible maximización (e.g. Azrin \& Lindsley, 1956; Bilbao \& Fernández, 1999; Camerer, 2003; Dugatkin, 1977; Glenn, 2004; Hake \& Olvera, 1978; Hake \& Vukelich, 1972; Hursh \& Roma, 2013; Homans, 1961; Okouchi, 2012; Osborne, 2004; Rostek, 2010; Shubik, 1982; Skinner, 1953, 1962; Stephens \& Anderson, 1997; Weiner, 1977; Weingarten \& Mechner, 1966; Yi \& Rachlin, 2004).

La suficiencia de tales aproximaciones para dar cuenta de fenómenos sociales ha sido cuestionada en más de un sentido. El Paradigma Interconductual (e.g. Kantor, 1929, 1936, 1982; Ribes, 2001; Ribes \& López, 1985; Ribes \& Rangel, 2002; Ribes, Rangel \& López, 2008; Ribes, 2010) y los trabajos de autores como Henrich y Henrich
(2007), Kropotkin (1902/2008), Pennisi (2005), Schuster y Perelberg (2004) o Wittgenstein (1953, 1958), constituyen tan sólo algunos ejemplos de posturas en las que el tratamiento de la conducta social no es reducido a la operación de variables meramente económicas. Se ha insistido, por ejemplo, en el papel fundamental del lenguaje en el análisis de la conducta social y, en especial, en el favorecimiento de la conducta cooperativa o, en términos de Ribes (2001), de la conducta altruista parcial (e.g. Pennisi, 2005; Ribes, Rangel, Magaña, López \& Zaragoza, 2005; Ribes et al., 2006; Ribes, Rangel, Ramírez et al., 2008; Wittgenstein, 1953, 1958). Ribes (2001) parte del supuesto de que el término cooperación es poco descriptivo y excluyente, en tanto que cooperar significa, en sentido estricto, operar en forma conjunta y, en esa medida, toda relación social es por definición cooperativa. En consecuencia, se consideró oportuno remplazar el término de cooperación por el término de altruismo parcial, empleado para describir una relación que implica dar y recibir, permitiendo con ello distinguir entre situaciones cooperativas de implicaciones tan distintas como la 
competencia o el altruismo. Es en atención a lo anterior que en el presente trabajo se adoptó el término altruismo parcial en sustitución del término cooperación.

Una colección importante de datos recabada por Ribes y colaboradores (Ribes, 2001; Ribes \& Rangel, 2002; Ribes, Rangel, Carbajal \& Peña, 2003; Ribes, Rangel, Casillas et al., 2003; Ribes, Rangel, Juárez et al., 2003) ha demostrado que la asignación de mayores ganancias asociadas a las respuestas dadas bajo una alternativa compartida de altruismo parcial respecto de las programadas por responder bajo una alternativa no compartida, resulta insuficiente para privilegiar la elección de la primera en arreglos experimentales con presentación concurrente de ambas alternativas. Resultados de trabajos posteriores condujeron a los autores a sostener que lo que parecía ser fundamental en el favorecimiento de la elección de la alternativa compartida de altruismo parcial respecto de la no compartida, era el establecimiento de intercambios verbales por parte de los participantes a modo de acuerdos para la resolución de la tarea (Ribes et al., 2005; Ribes et al., 2006; Ribes, Rangel, Ramírez et al., 2008). La afirmación de Pennisi (2005) en el sentido de que “... las interacciones lingüísticas explícitas pueden ser una condición necesaria para el desarrollo de conducta bajo contingencias compartidas" (p.93) parece suscribir dichos asertos.

Hallazgos y afirmaciones como las anteriores permiten establecer el siguiente supuesto general: que el intercambio verbal favorece la elección de alternativas de respuesta compartidas (e.g. Pennisi, 2005; Ribes et al., 2005; Ribes et al., 2006; Ribes, Rangel, Ramírez et al., 2008). Sin embargo, resta aún el estudio sistemático de las condiciones o circunstancias que favorecen que el establecimiento de intercambios verbales de cierta cualidad funcional a dichos fines tenga lugar (i.e. acuerdos para la resolución de la tarea).

Dado que la conducta social se da siempre como conducta en situación, su estudio no puede realizarse al margen de las condiciones o circunstancias en las cuales tiene lugar. Dichas condiciones o circunstancias pueden ser analizadas y acotadas acudiendo al concepto de contingencia que, empleado para describir relaciones de condicionalidad, circunstancialidad o dependencia, comprende a las relaciones de ocurrencia y de función que definen a cualquier evento psicológico (Ribes, 2001, 2010; Ribes et al. 2008a).

Así, en tanto que cada individuo ejerce diferentes funciones en diferentes ámbitos institucionales es posible decir que "...sus características sociales son siempre contingentes, es decir, circunstanciales a dichos ámbitos" (Ribes, Rangel \& López, 2008, p. 48). Más aún, las instituciones mismas pueden ser caracterizadas como sistemas de contingencias sociales que regulan las interacciones entre individuos.

En concordancia con lo anterior, en un estudio previo Pulido, Rangel y Ortiz (2013), analizaron el papel del intercambio verbal en la solución de tareas encontrando que la cualidad predominante (en términos de su contenido referido a propiedades particulares, generales o relacionales de la tarea) de los intercambios verbales que los participantes establecieron, varió en función del tipo de contingencia a la que fueron expuestos (i.e. contingencias individuales, colectivas o compartidas). Además de corroborar la relación existente entre el tipo de contingencia y la cualidad de los intercambios verbales ya antes reportada, el actual estudio permitió estimar el impacto de dichas variables en la conducta de elección por contingencias no compartidas o por contingencias compartidas de altruismo parcial, toda vez que los datos de estudios previos (Pulido et al., 2013; Ribes et al., 2005; Ribes et al., 2006; Ribes, Rangel, Ramírez et al., 2008) nos permiten suponer que no es el mero establecimiento de intercambios verbales lo que inclina la preferencia por alternativas compartidas de altruismo parcial, sino el establecimiento de cierta cualidad de intercambios verbales (i.e. establecimiento de acuerdos) y no otra, cuya emergencia es circunstancial y, por lo tanto, se relaciona con la organización contingencial vigente en cada caso.

De acuerdo con lo antes expuesto, con el presente experimento se pretendió explorar de manera sistemática el papel de la exposición diferencial a distintas secuencias de arreglos contingenciales en el establecimiento y la cualidad 
de intercambios verbales referidos a la tarea y como estos, a su vez, intervienen en la conducta de elección de contingencias no compartidas y/o compartidas de altruismo parcial en niños estudiantes de primaria.

A tal fin, se recurrió a la manipulación como variable independiente de la secuencia de exposición en general y del tipo de contingencia en particular a la que los participantes fueron expuestos. Dentro de esta propuesta, se consideraron dos tipos contingencias principales como el contexto o circunstancia que enmarca la funcionalidad de los intercambios verbales y de la conducta de elección, esta última relativa a las contingencias compartidas: contingencias no compartidas (mismas que podían ser de tipo individual o colectivo) y contingencias compartidas, para acortar su denominación (mismas que presentaban dos alternativas de respuesta, una individual o no compartida y una compartida de altruismo parcial), tal y como fueron empleadas en los estudios de Ribes y colaboradores a propósito de diversos parámetros de la conducta social (e.g. Ribes \& Rangel, 2002; Ribes, Rangel, Carbajal et al., 2003; Ribes, Rangel, Casillas et al., 2003; Ribes, Rangel, Juárez et al., 2003).

\section{Método}

\section{Participantes}

Participaron voluntariamente 36 estudiantes de sexto grado de primaria (18 niños y 18 niñas), de entre los 11 y los 13 años de edad, a cambio de premios (artículos de papelería, juguetes o artículos de arreglo personal), que se les mostraron al inicio de cada sesión con su respectivo valor en puntos. Los participantes fueron distribuidos al azar en 18 díadas experimentales conformadas por niños del mismo sexo, ya sea en un arreglo niño-niño o niña-niña en aras de evitar posibles efectos de inhibición lingüística.

\section{Aparatos y tarea experimental}

Se utilizó una tarea de armado de rompecabezas virtuales. Para las fases individuales la tarea se realizó en dos computadoras independientes. Por su parte, para las fases colectivas y compartidas la tarea se realizó en dos computadoras que estuvieron interconectadas en red.

Las respuestas de los participantes fueron registradas de manera automática por el programa. Todas las computadoras estuvieron equipadas con un procesador Pentium-IV, monitor cromático, teclado y controles de juego para responder. El software fue diseñado en el programa Blitz Max v1.26. Las instrucciones y la tarea (rompecabezas) se presentaron en la computadora. Los datos se analizaron con el programa PASW Statistics versión 17.0 y se graficaron con Sigma Plot versión 11.0. Las interacciones verbales fueron registradas empleando una grabadora de voz digital.

\section{Situación experimental}

Las sesiones se llevaron a cabo en uno o dos salones de clases de una escuela primaria. El primer día se realizó una sesión de demostración y las tres sesiones experimentales correspondientes a la Fase 1. Para el segundo y tercer día se pusieron en marcha las tres sesiones experimentales correspondientes a la Fase 2 y a la Fase 3 respectivamente.

Los participantes trabajaron en un mismo salón en la condición de participantes juntos (cuatro primeras díadas de cada grupo experimental) y en salones distintos en la condición de participantes separados (dos últimas díadas de cada grupo experimental). Se colocó una grabadora de voz en el cubículo experimental para registrar las interacciones verbales entabladas entre los participantes de las díadas asignadas a la condición juntos de cada grupo. Los salones experimentales contaron con una superficie aproximada de 12 metros cuadrados y se hallaron libres de ruidos y distractores.

\section{Diseño experimental}

Para el presente experimento se utilizó un diseño intrasujeto con comparación entre sujetos bajo el mismo y bajo distintos tratamientos experimentales (ver Tabla 1). Los participantes se distribuyeron al azar en tres grupos experimentales de doce integrantes cada uno (seis díadas por grupo). Las cuatro primeras díadas de cada grupo fueron asignadas a la condición juntos (con ambos participantes 
en la misma sala experimental), mientras que las dos últimas díadas fueron asignadas a la condición separados (con un participante por sala experimental).

Previo a las fases experimentales, todos los participantes fueron entrenados en el uso del programa computacional que presentó la tarea experimental. Concluida esta sesión de demostración, las díadas fueron expuestas a tres fases experimentales compuestas por 3 sesiones cada una (se presentaron tres rompecabezas distintos por fase). La Fase 1 se desarrolló bajo las directrices de la contingencia No Compartida Individual, esto con afán de evaluar el efecto de la exposición inicial ante tal tipo de contingencia en la conducta de elección por contingencias compartidas/no compartidas programada en fases posteriores.

Después de la Fase 1, los participantes fueron expuestos a dos fases experimentales, diferenciales según el grupo. Para el Grupo 1, la Fase 2 se rigió por los parámetros marcados para la contingencia No Compartida Individual, mientras que la Fase 3 se desarrolló siguiendo las directrices de la contingencia Compartida. En tanto se refiere al Grupo 2, la Fase 2 se dirigió de acuerdo a los parámetros de la contingencia No Compartida Colectiva, mientras que la Fase 3 se desarrolló según marca la contingencia Compartida. Por último, en el Grupo 3, tanto la Fase 2 como la Fase 3 se programaron según los parámetros de la contingencia Compartida.

Tabla 1. Muestra el Diseño Experimental.

\begin{tabular}{|c|c|c|c|c|c|}
\hline & & & Fase 1 & Fase 2 & Fase 3 \\
\hline \multirow{2}{*}{ Grupo 1} & $\begin{array}{l}\text { Juntos } \\
\mathrm{N}=4 \mathrm{~d}\end{array}$ & $\begin{array}{l}\mathrm{D} \\
\mathrm{e}\end{array}$ & \multirow{6}{*}{$\begin{array}{l}\text { No Compartida } \\
\text { (Individual) }\end{array}$} & \multirow{2}{*}{$\begin{array}{l}\text { No Compartida } \\
\text { (Individual) }\end{array}$} & \multirow{2}{*}{ Compartida } \\
\hline & $\begin{array}{l}\text { Separados } \\
N=2 d\end{array}$ & \multirow{2}{*}{$\begin{array}{l}\mathrm{m} \\
\mathrm{O} \\
\mathrm{s}\end{array}$} & & & \\
\hline \multirow{2}{*}{ Grupo 2} & $\begin{array}{l}\text { Juntos } \\
\mathrm{N}=4 \mathrm{~d}\end{array}$ & & & \multirow{2}{*}{$\begin{array}{l}\text { No Compartida } \\
\text { (Individual) }\end{array}$} & \multirow{2}{*}{ Compartida } \\
\hline & $\begin{array}{l}\text { Separados } \\
\mathrm{N}=2 \mathrm{~d}\end{array}$ & \multirow{3}{*}{$\begin{array}{l}\mathrm{r} \\
\mathrm{a} \\
\mathrm{c} \\
\mathrm{i} \\
\mathrm{o} \\
\mathrm{n}\end{array}$} & & & \\
\hline \multirow{2}{*}{ Grupo 3} & $\begin{array}{l}\text { Juntos } \\
\mathrm{N}=4 \mathrm{~d}\end{array}$ & & & \multirow{2}{*}{$\begin{array}{l}\text { No Compartida } \\
\text { (Individual) }\end{array}$} & \multirow{2}{*}{ Compartida } \\
\hline & $\begin{array}{l}\text { Separados } \\
N=2 d\end{array}$ & & & & \\
\hline Sesiones & & 1 & 3 & 3 & 3 \\
\hline Días & & $1^{\circ}$ & $1^{\circ}$ & $2^{\circ}$ & $3^{\circ}$ \\
\hline
\end{tabular}

\section{Procedimiento}

La tarea experimental consistió en el armado de rompecabezas virtuales a través de una computadora. La pantalla mostró rompecabezas de 50 piezas para las condiciones individuales, colectivas y compartidas. Se utilizó un modelo de rompecabezas distinto para cada sesión con el fin de evitar efectos de aprendizaje. Los participantes pudieron ver la figura del rompecabezas que armarían en la sesión durante un minuto, posteriormente esta desapareció y se desplegó un cursor en forma de manita que sirvió a los participantes para la selección y colocación de las piezas del rompecabezas (ver figuras 1 y 2). Los participantes tuvieron un tiempo límite de cinco minutos (adicionales al minuto inicial de visualización de la imagen del rompecabezas en turno) para resolver cada uno de los rompecabezas a fin de evitar posibles efectos de fatiga por la exposición prolongada a la tarea experimental.

La clasificación de los intercambios verbales establecidos por los participantes de las díadas 
asignadas a la condición juntos se realizó atendiendo a su cualidad en términos de contenido con base en tres categorías principales:

a) Intercambios verbales particulares. Se refieren a propiedades específicas de piezas particulares. b) Intercambios verbales generales. Se refieren a propiedades específicas de grupos de piezas.

c) Intercambios verbales estratégicos. Se refieren al establecimiento de acuerdos o estrategias conjuntas para la resolución de la tarea (ver Tabla 2).

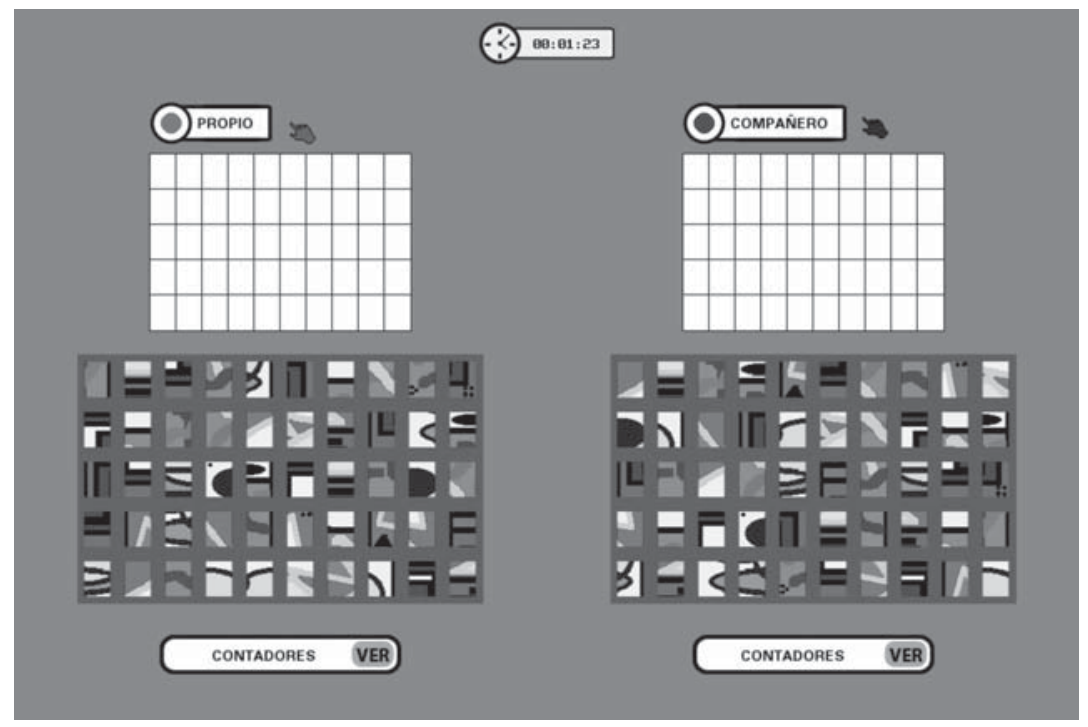

Figura 1. Muestra el arreglo típico de las condiciones no compartida colectiva y compartida, para la condición no compartida individual se presentó un solo rompecabezas en pantalla.

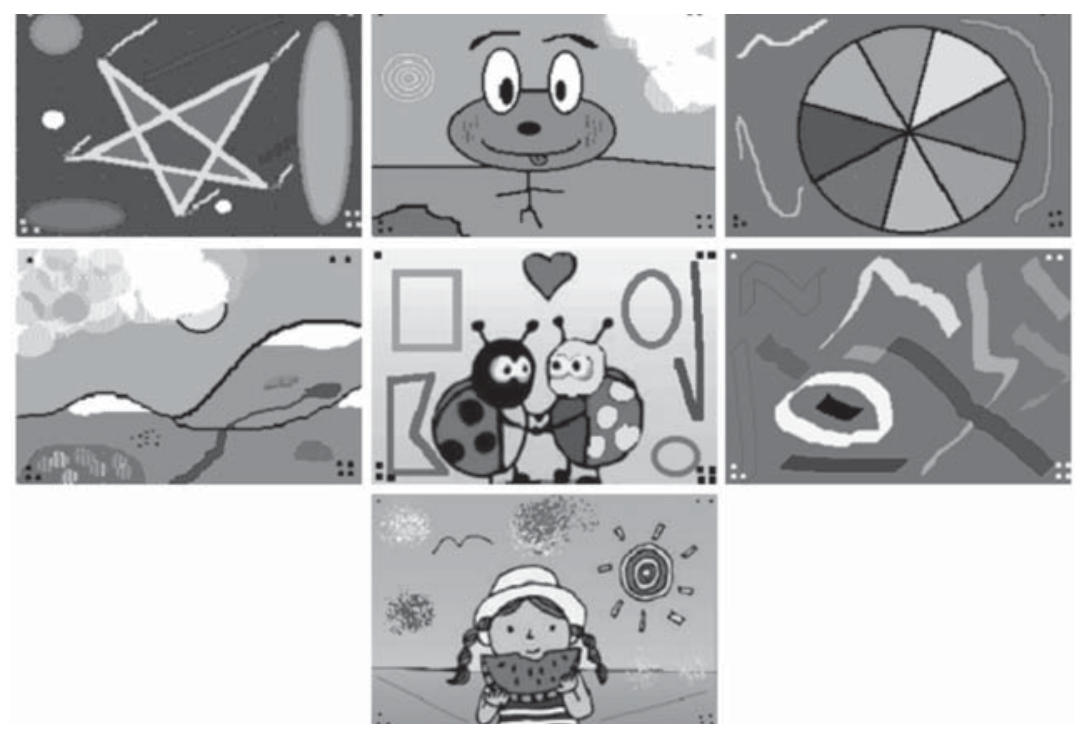

Figura 2. Muestra ejemplos de las imágenes de los rompecabezas de 50 piezas usados en este experimento. 
Tabla 2. Muestra ejemplos de los intercambios verbales establecidos por los participantes de distintas díadas y su clasificación de acuerdo a su cualidad particular, general o estratégica.

\begin{tabular}{lll}
\hline \multicolumn{1}{c}{ Particulares } & \multicolumn{1}{c}{ Generales } & \multicolumn{1}{c}{ Estratégicos } \\
\hline P8: Esta no queda en ningún lado. & P7: A mí me gusta irme por filas, & P7: ¿Tú en el mío y yo en el tuyo? \\
P7: ¿Cuál? & así no me pierdo, primero todas las & P8: Si, pero si te sabes una que \\
P8: La de la mancha rosa. & verdes y luego las azules. & yo no he puesto me dices para \\
P7: Si queda, yo ya la puse. & P8: Yo pongo las orillas primero & ponerla. \\
& porque están más fáciles. & P7: Órale, tú también. Hay que \\
& ayudarnos en todas. \\
& P8: O hay que ir poniendo las \\
& mismas. \\
& P7: No, mejor cada vez que \\
& pongamos una nos decimos dónde \\
& va. \\
& P8: Bueno, pero rápido para \\
& ganarnos muchos premios.
\end{tabular}

P14: ¿Ya encontraste la de la boquita?

P13: $\mathrm{Si}$, pero primero puse el ojo para ubicarme dónde iba.
P13: Este está difícil, tiene muchas piezas iguales P14: Si, muchas blancas y verdes. P13: Yo primero voy a poner todas las verdes.
P14: Tú apréndete la parte de la izquierda y yo la de la derecha, ¿sale?

P13: Bueno. Cuando lo estemos armando tú pones la parte de la izquierda primero en mí rompecabezas y yo pongo la parte de la derecha en tu rompecabezas para tener modelo de cómo se hace. P14: Bueno, pero si no sé dónde va una pieza me dices ¿sí? P13: Si, tú también
P27: ¿Ya pusiste la blanca? P28: No

P27: No encuentro en dónde va.
P27: Las piezas de las esquinas son mis favoritas porque tienen pistas. P28: Si, son las más fáciles, esas y las de la figura.
P27: ¿Cómo le hacemos?

P28: Hay que empezar a armar de afuera hacia adentro.

P27: Bueno, ¿tú armas mi rompecabezas y yo el tuyo verdad? P28: Si
La confiabilidad de este análisis se realizó por consenso entre dos observadores (100\%).

\section{Demostración}

Como se mencionó, al inicio de la tarea se instruyó a los participantes en el uso del software computacional mediante una demostración interactiva, tipo tutorial, en la cual se mostraron las distintas funciones del programa de armado de rompecabezas (i.e. cómo seleccionar, mover y colocar las piezas del rompecabezas, la identificación de aciertos o errores en su ejecución).

Contingencia No Compartida Individual

La contingencia No Compartida Individual consistió en la resolución individual de un rompecabezas de 50 piezas, sin posibilidad de ver la ejecución del compañero. Los participantes fueron colocados en el mismo cubículo o en cubículos separados según correspondiera a la condición 
implicada y se asignó una computadora con su respectivo control de mando por cada participante. En la pantalla de la computadora se desplegó un cursor sobre las piezas del rompecabezas, mismo que el participante pudo mover con el control respectivo. El participante debió colocar el cursor sobre la pieza que deseaba mover y además presionar el botón 1 para seleccionar la pieza; para activar el movimiento de la pieza debió hacer uso de las flechas direccionales del control (hacia arriba, abajo, izquierda y derecha) y para colocar la pieza en el lugar deseado debió seleccionar dicho lugar con el cursor y presionar de nueva cuenta el botón 1. Por cada pieza colocada el participante obtuvo 10 puntos. Al final de cada fase, los participantes recibieron los premios correspondientes a la puntuación alcanzada con su ejecución.

\section{Contingencia No Compartida Colectiva}

La contingencia No Compartida Colectiva consistió en la resolución individual de un rompecabezas de 50 piezas, con la visualización en pantalla de la ejecución del compañero a través de la interconexión en tiempo real de dos computadoras. Se asignó una computadora por integrante, y los participantes resolvieron la tarea en el mismo o en diferentes cubículos experimentales según correspondiera a la condición en curso. Los participantes solamente pudieron responder en su propio rompecabezas, sin embargo, tuvieron la posibilidad observar en pantalla su desempeño y el del compañero (la mitad izquierda de la pantalla mostró el rompecabezas propio y la mitad derecha mostró el rompecabezas del compañero). La operación del programa fue idéntica a la de las sesiones bajo la contingencia no compartida individual. Por cada pieza colocada el participante obtuvo 10 puntos. Al final de cada fase, los participantes recibieron los premios correspondientes a la puntuación alcanzada con su ejecución.

\section{Contingencia Compartida}

La contingencia Compartida presentó dos alternativas de respuesta de manera concurrente: una no compartida o individual y otra compartida o social de altruismo parcial, esto es, el participante tuvo la opción de responder en su propio rompecabezas (alternativa no compartida), en el rompecabezas del compañero (alternativa compartida) o en ambos. En esta fase se empleó un sistema de intercambio de altruismo parcial, tal y como ha sido definido en estudios de Ribes y colaboradores (e.g. Ribes \& Rangel, 2002; Ribes, Rangel, Juárez et al., 2003; Ribes et al., 2005) en donde las piezas colocadas en el rompecabezas individual generaron puntos solo para el participante que las colocó (10 puntos), mientras que las colocadas en el rompecabezas del compañero retribuyeron puntos iguales para ambos participantes (10 puntos para cada uno).

\section{Resultados}

La Figura 3 muestra la duración total de las interacciones verbales no referidas y referidas a la tarea y su cualidad particular, general o estratégica de los Grupos 1 al 3 de la condición juntos por cada una de las fases experimentales.

Para el Grupo 1 (expuesto a las fases no compartida individual-no compartida individual-compartida) se observó, en general, que la cualidad de intercambios verbales que los participantes establecieron fue predominantemente particular durante las dos primeras fases experimentales. Sin embargo, al introducirse la Fase 3, de tipo compartida, aumentó el tiempo total de conversación referido a la tarea (pasando de un promedio por grupo de 2.29 minutos en la Fase 1 a un promedio por grupo de 5.38 minutos en la Fase 3). Además, durante esta fase, la cualidad de los intercambios verbales se volvió predominantemente estratégica. Destaca el caso de la Díada 3 de este grupo experimental, cuyos participantes permanecieron en silencio prácticamente durante todas las fases experimentales.

Los datos de la Fase 1 del Grupo 2 (expuesto a las fases no compartida individual-no compartida colectiva-compartida) muestran que los intercambios verbales ahí presentados fueron de cualidad exclusivamente particular. Al introducirse la Fase 2, la cualidad de los intercambios verbales se volvió predominantemente general y aumentó el tiempo de conversación total referido a la tarea (pasando de $1.98 \mathrm{mi}$ nutos promedio por grupo en la Fase 1 a un promedio por grupo de 3.86 minutos durante 
la Fase 2). Ya para la Fase 3, de tipo compartida, los intercambios verbales alcanzaron las mayores duraciones de del grupo (5.8 minutos en promedio por grupo) y la cualidad de dichos intercambios se tornó principalmente estraté- gica. Aquí, es la Díada 10 el caso de excepción, toda vez que se observó que los intercambios verbales establecidos por los participantes fueron mínimos y, en todo caso, su contenido fue ajeno a la tarea experimental.

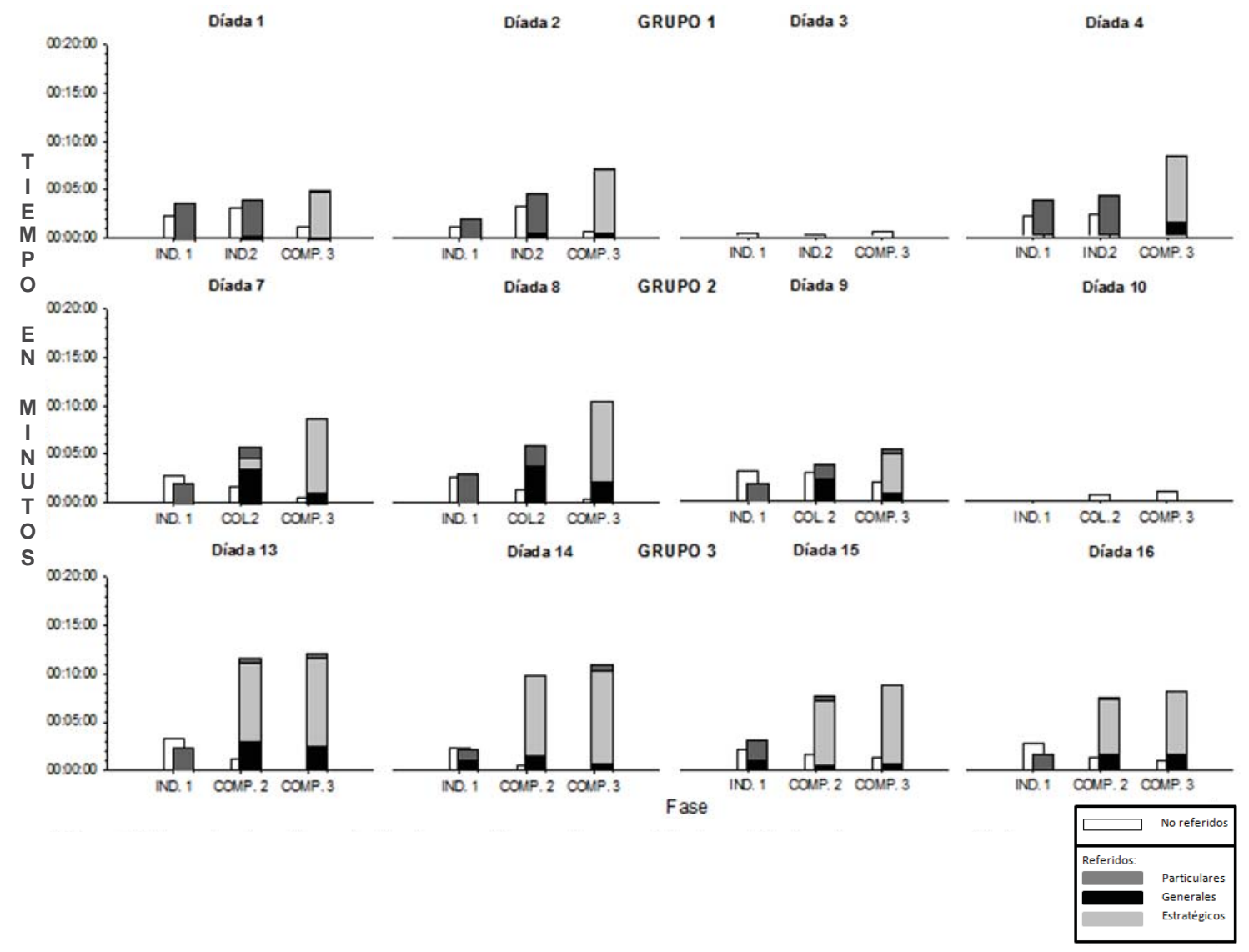

Figura 3. Muestra la duración total de los intercambios verbales no referidos y referidos a la tarea y su cualidad particular, general o estratégica de los grupos 1 al 3 de la condición juntos por cada una de las fases experimentales.

En el Grupo 3 (expuesto a las fases no compartida individual-compartida-compartida), se encontró que los intercambios verbales referidos a la tarea fueron de cualidad predominantemente particular y de breve duración (2.33 minutos en promedio por grupo) en la Fase 1. Sin embargo, al introducirse la Fase 2 se observó un aumento en el tiempo de conversación referido a la tarea (pasando de un promedio por grupo de 2.33 minutos para la Fase 1 a un promedio por grupo de 8.57 minutos en la Fase 2). Asimismo, a partir de la Fase 2 se diversificó la cualidad de los intercambios verbales, Ilegando a ser mayoritariamente de tipo estratégico además de los particulares y generales presentes desde la fase inmediatamente anterior. Por último, al introducirse la Fase 3 se mantuvo la tendencia en el aumento y predominancia de las interacciones de tipo estratégico, además, se alcanzó el valor de tiempo de conversación referido a la tarea más alto de todo el experimento (13.6 minutos en promedio por grupo). 
La Figura 4 (panel A), muestra el promedio de piezas colocadas correctamente en cada fase de la condición juntos del Grupo 1 (no compartida individual-no compartida individualcompartida). Como se ha visto, durante las contingencias compartidas los participantes pudieron elegir entre contestar en el rompecabezas local (alternativa no compartida) y/o el rompecabezas remoto (alternativa compartida de altruismo parcial), mientras que durante las fases no compartidas (individuales o colectivas) los participantes sólo pudieron responder en el rompecabezas local. En general, puede observarse que al introducirse la contingencia compartida en la Fase 3, todas las díadas que establecieron intercambios verbales referi- dos a la tarea (díadas 1, 2 y 4) prefirieron responder predominantemente en la alternativa compartida. En cambio, los participantes de la Díada 3, mismos que permanecieron en silencio prácticamente durante todas las fases experimentales, optaron por responder exclusivamente en la alternativa no compartida (ver Figura 4, panel A).

La Figura 4 (panel B) muestra el promedio de piezas colocadas de manera correcta en cada fase de la condición separados del Grupo 1 (no compartida individual-no compartida individualcompartida). Los datos muestran que ambas díadas permanecieron respondiendo predominantemente en la alternativa no compartida durante la Fase 3, de tipo compartida.

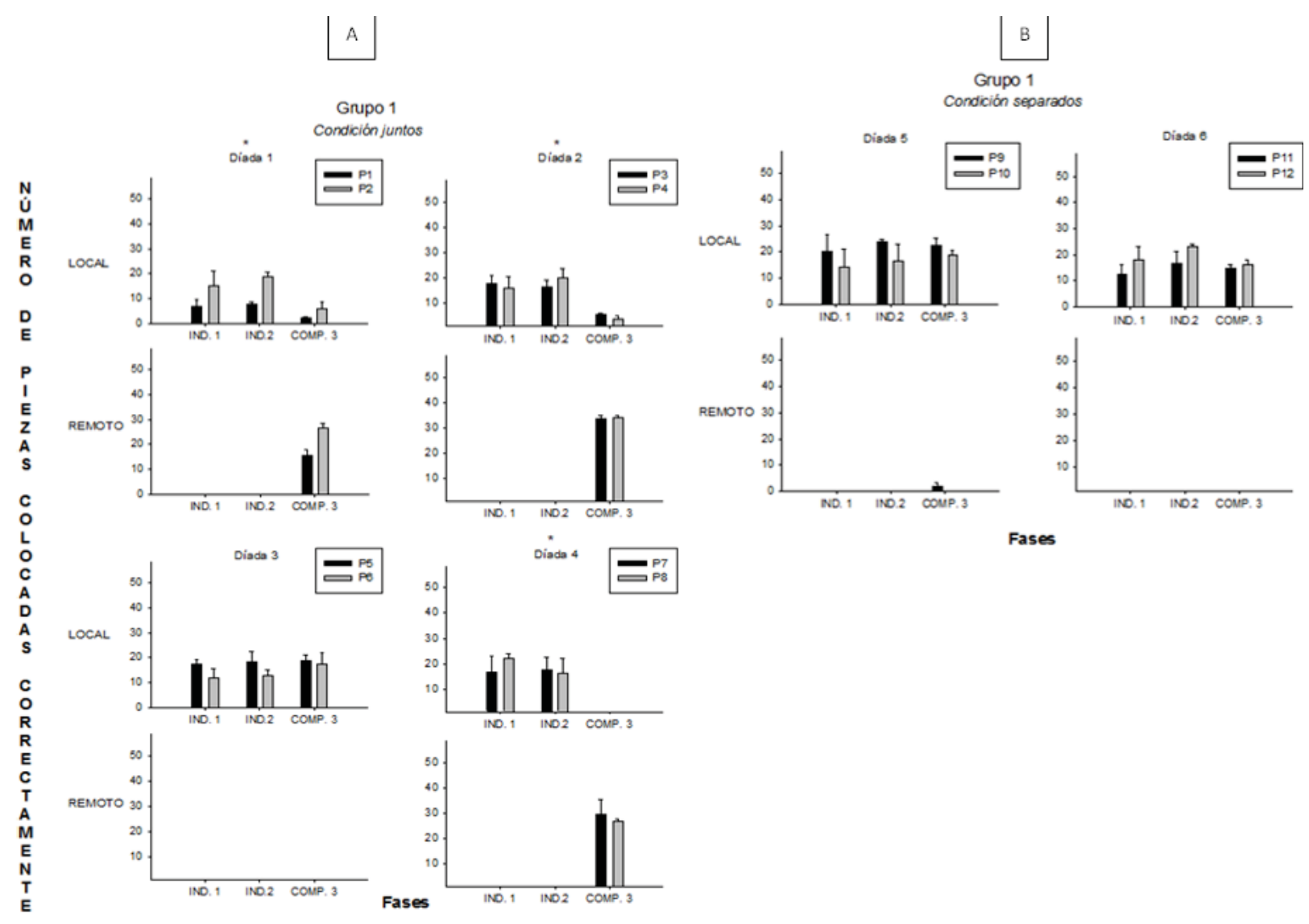

Figura 4. Muestra el promedio de piezas colocadas correctamente por cada fase de la condición juntos (A) y separados (B) del Grupo 1. 
La Figura 5 (panel A) muestra el promedio de piezas colocadas correctamente en cada fase de la condición juntos del Grupo 2 (no compartida individual-no compartida colectivacompartida). Se observa que durante la Fase 3 de tipo compartida, a excepción de la Díada 10, cuyos participantes optaron por permanecer en silencio durante la mayor parte del experimento, todos los participantes prefirieron responder en la alternativa compartida. Este dato es apoya lo observado en el Grupo 1 (ver Figura 4, panel A).
La Figura 5 (panel B) muestra el promedio de piezas colocadas de manera correcta en cada fase de la condición separados del Grupo 2 (no compartida individual-no compartida colectiva-compartida). Aquí, puede observarse, de manera consistente con los datos reportados anteriormente, que los participantes en aislamiento verbal del presente grupo se mantuvieron contestando de manera casi exclusiva en la contingencia no compartida durante la Fase 3.

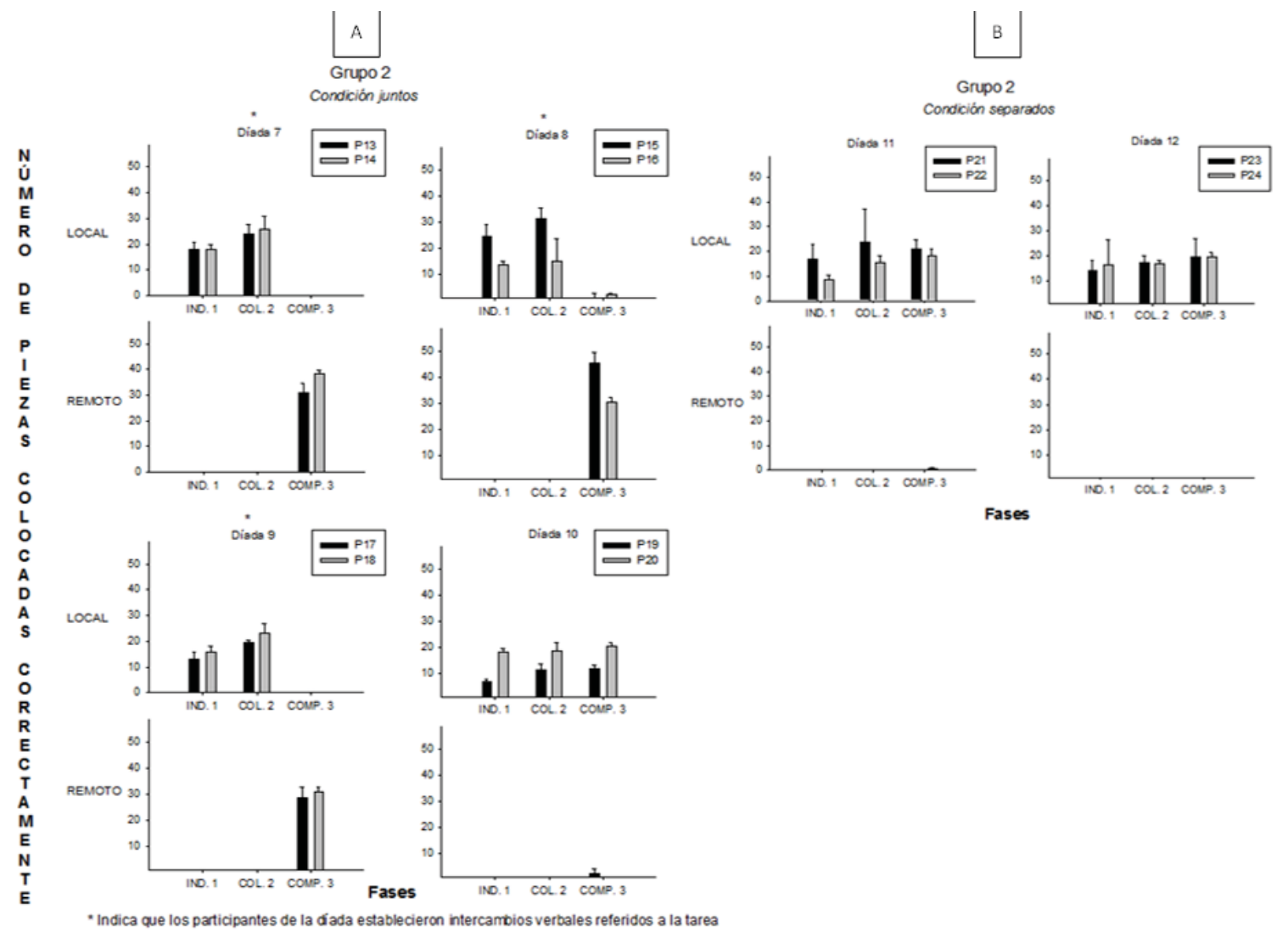

Figura 5. Muestra el promedio de piezas colocadas correctamente por cada fase de la condición juntos (A) y separados (B) del Grupo 2.

La Figura 6 (panel A) muestra el promedio de piezas colocadas correctamente en cada fase de la condición juntos del Grupo 3 (no compartida individual-compartida-compartida). Se aprecia que apenas introducida la contingencia compartida (Fase 2), los participantes respondieron casi de manera exclusiva en la alternativa compartida (los participantes colocaron apenas un promedio de 0.7375 piezas en la alternativa no compartida), hecho que coincide en todos los casos con el establecimiento de acuerdos verbales de cualidad estratégica (i.e. entonces tú respondes solo en mi rompecabezas y yo en el tuyo para ganar todos los puntos). 
La Figura 6 (panel B) muestra el promedio de piezas colocadas de manera correcta en cada fase de la condición separados del Grupo 3 (no compartida individual-compartida-compartida). Aquí, se observa que todos los participantes prefirieron responder en la alternativa no compartida sobre la compartida, en tal sentido que los participantes colocaron apenas un promedio de 0.675 piezas en la alternativa compartida durante las fases de elección (fases 2 y 3 ).

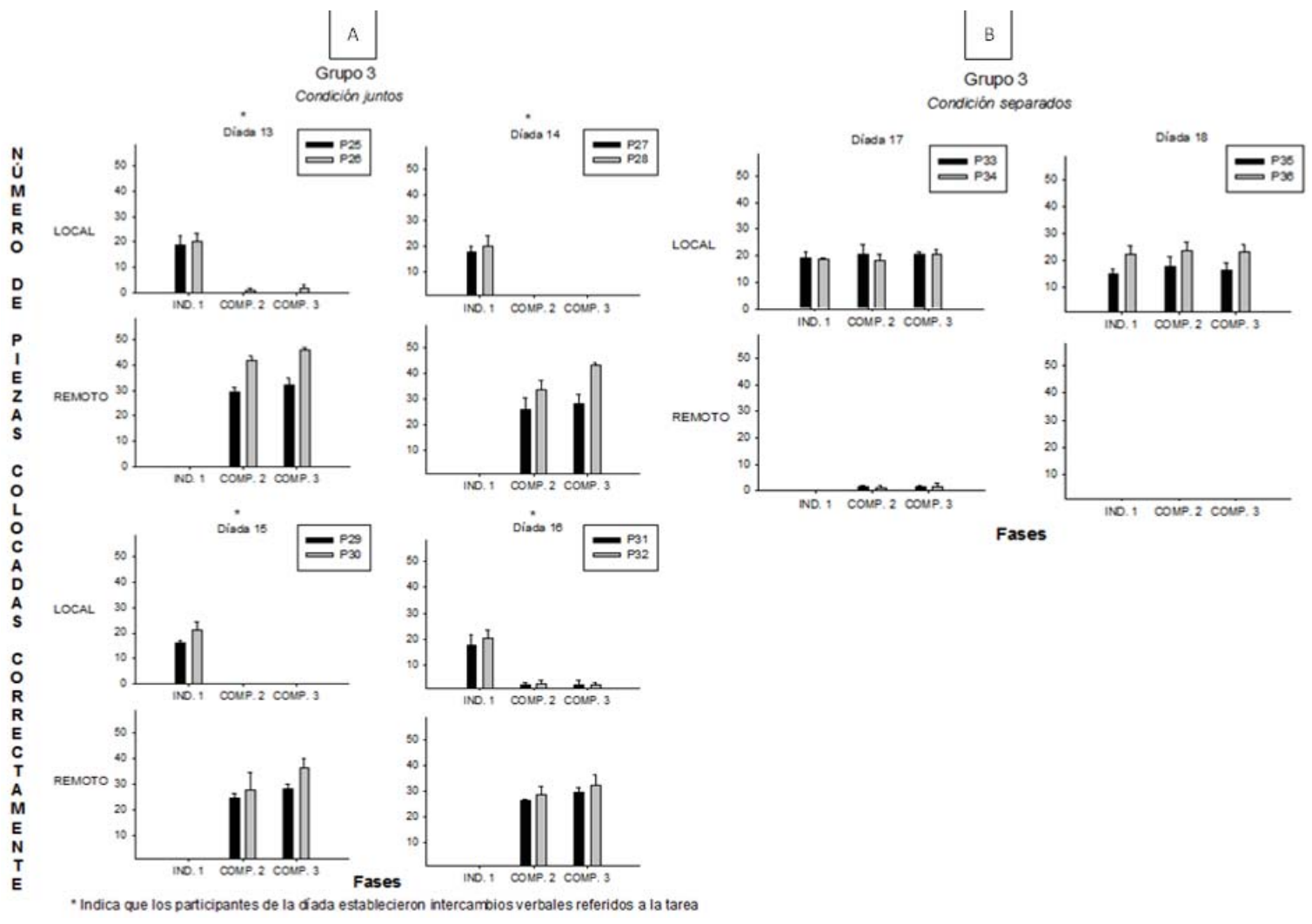

Figura 6. Muestra el promedio de piezas colocadas correctamente por cada fase de la condición juntos (A) y separados (B) del Grupo 3.

\section{Discusión}

Los resultados obtenidos dan cuenta de dos hallazgos fundamentales. El primero, apoya lo encontrado en el estudio de Pulido et al. (2013) al sugerir, en general, que el tipo de contingencia a la que los participantes son expuestos se relaciona de manera directa con la cualidad predominante de los intercambios verbales que ellos mismos establecen; así, las contingencias no compartidas individuales parecen relacionarse con el establecimiento de intercambios de cualidad predominantemente particular, las contingencias no compartidas colectivas con una cualidad predominantemente general y las contingencias compartidas con una cualidad predominantemente estratégica.

Que la cualidad predominante de los intercambios verbales varíe en función del tipo de contingencia en turno permite suponer, en principio, que el reconocimiento de la organización contingencial vigente en cada caso contribuye a la delimitación de la pertinencia funcional de los intercambios verbales. Cada tipo de contingencia (i.e. compartidas y no compartidas) entraña ciertas propiedades morfológicas y funcionales distinti- 
vas que determinan los criterios de pertinencia específicos a esta. La cualidad predominante de los intercambios verbales corresponde entonces a tales criterios de pertinencia (Ribes, Rangel, \& López, 2008).

Así, por ejemplo, el establecimiento de intercambios verbales de cualidad predominantemente estratégica (i.e. acuerdos para la resolución de la tarea) durante las contingencias compartidas es pertinente en tanto que las condiciones dadas para la resolución de la tarea bajo dichas contingencias implican la posibilidad de intervenir directamente en la tarea del otro participante, por lo que acordar las condiciones o estrategias y las implicaciones de dicha intervención cobra sentido. Dado que la posibilidad de intervenir en la tarea del otro se encuentra restringida durante las contingencias no compartidas, la cualidad resultante de los intercambios verbales predominantes pertinentes es otra, una más dirigida a la descripción de propiedades particulares o generales de los elementos constitutivos de la tarea.

El segundo hallazgo concuerda con los reportes de Ribes y colaboradores (Ribes et al., 2005; Ribes et al., 2006; Ribes, Rangel, Ramírez et al., 2008) y los postulados de Pennisi (2005) al apuntar a que el establecimiento de intercambios verbales resulta crucial en el favorecimiento de la elección de alternativas compartidas de altruismo parcial sobre las no compartidas.

Como se observó, todos los participantes que establecieron intercambios verbales, cuya cualidad fue predominantemente estratégica, durante la fase de elección entre una alternativa de respuesta no compartida y una alternativa de respuesta compartida de altruismo parcial (contingencia compartida), prefirieron responder en la alternativa compartida, mientras que los participantes que no establecieron intercambios verbales referidos a la tarea por decisión (díadas 3 y 10 de la condición de participantes juntos) o por la restricción característica de la condición experimental (todas las díadas asignadas a la condición de participantes separados), se mantuvieron respondiendo sistemáticamente en la alternativa no compartida.

Como los casos de la díadas 3 y 10 demuestran, cuyos participantes sólo establecieron intercambios verbales no referidos a la tarea experimental, no es suficiente el mero establecimiento de intercambios verbales para favorecer la elección de contingencias compartidas de altruismo parcial, sino el establecimiento de intercambios verbales de cierta cualidad funcional a dichos fines (i.e. acuerdos o estrategias para la resolución de la tarea).

Aunado a lo anterior, los participantes eligieron responder en la alternativa compartida de altruismo parcial siempre que establecieron intercambios verbales de cualidad estratégica, pese a ser expuestos inicialmente ante contingencias no compartidas individuales. La exposición inicial ante contingencias no compartidas individuales no parece determinar, por tanto, la ejecución en fases posteriores, sino la ausencia o el establecimiento de intercambios verbales de cierta cualidad funcional a los requerimientos de la contingencia vigente.

Dado que se observó que todos los participantes que no establecieron intercambios verbales de cualidad estratégica permanecieron respondiendo en la alternativa no compartida, pese a que dicha alternativa de respuesta otorgaba la mitad de las ganancias posibles en relación con las ganancias disponibles por responder en la alternativa compartida, puede decirse que la tesis económica que intenta explicar la conducta social dictada desde constructos teórico-experimentales como el Paradigma Operante del Análisis Experimental de la Conducta (e.g. Azrin \& Lindsley, 1956; Glenn, 2004; Hake \& Vukelich, 1972; Hursh \& Roma, 2013; Lindsley, 1966; Okouchi, 2012; Skinner, 1962; Weiner, 1977; Yi \& Rachlin, 2004) o la Teoría de Juegos (e.g. Bilbao \& Fernández, 1999; Camerer, 2003; Gibbons, 1997; Osborne, 2004; Rostek, 2010; Shubik, 1982) y que puede resumirse para fines prácticos en un enunciado tal como las consecuencias y su posible maximización son suficientes para promover la conducta cooperativa, no ha sido apoyada por los datos recabados en este experimento.

En síntesis, los resultados obtenidos en este estudio parecen indicar que a) la cualidad de los intercambios verbales que los participantes establecen se relaciona con el tipo de contingencia operativa y b) que el establecimiento de intercambios verbales de cualidad estratégica se 
relaciona con la elección de alternativas sociales o compartidas de altruismo parcial sobre las individuales o no compartidas.

Que el intercambio verbal juegue un papel protagónico en la definición de los fenómenos sociales no resulta en absoluto sorprendente. Tiempo atrás autores como Weiss (1926), Wittgenstein (1953) o Ribes (2001) habían destacado su importancia. Weiss (1926), por ejemplo, sostuvo que la cooperación entre individuos descansa en la especialización del trabajo, la comunicación y el intercambio de capacidades y estrategias entre individuos. Pero los intercambios verbales no se dan el vacío, y es la aportación fundamental del presente trabajo sugerir que están relacionados con la organización contingencial que opera en cada una de las situaciones y sin la cual carecían de sentido.

Probar si hallazgos como estos pueden ser replicados en poblaciones con distintos grados de instrucción académica y mayor experiencia lingüística (Kantor, 1936), explorar el efecto de la variación en la secuencia de presentación de las contingencias y la prueba de otros tipos de contingencias bajo otros tipos de sistemas de intercambio sobre el establecimiento de intercambios verbales por parte de los participantes, son tan sólo algunas de las perspectivas de investigación que se abren a partir de los resultados obtenidos en el presente trabajo.

\section{Referencias}

Azrin, N., \& Lindsley, O. (1956). The reinforcement of cooperation between children. Journal of Abnormal and Social Psychology, 52, 100102. doi:10.1037/h0042490

Bilbao, J. M., \& Fernández, F. R. (Eds.) (1999). Avances en teoría de juegos con aplicaciones económicas y sociales. Sevilla, España: Universidad de Sevilla.

Camerer, C. F. (2003). Behavioral Game Theory: Experiments on strategic interaction. New Jersey, NJ: Princeton University Press.

Dugatkin, L. A. (1977). Partner choice, game theory and social behavior. Journal of Quantitative Anthropology, 5, 3-14.

Gibbons R. (1997). An introduction to applicable Game Theory. The Journal of Economic
Perspectives, 11, 127-149. doi:10.1257/ jep.11.1.127

Glenn, S. (2004). Individual behavior, culture, and social change. The Behavior Analyst, 27, 133151.

Hake, D. F., \& Olvera, D. (1978). Cooperation, competition, and related social phenomena. En T. A. Brigham, \& A. C. Catania (Eds.), Handbook of applied behavior analysis: Social and instructional processes (pp. 208245). New York, NY: Irvington.

Hake, D. F., \& Vukelich, R. (1972). A classification and review of cooperation procedures. Journal of the Experimental Analysis of Behavior, 18, 333-343. doi:10.1901/jeab.1972.18-333

Henrich, N., \& Henrich, J. (2007). Why humans cooperate. A cultural and evolutionary explanation. New York, NY: Oxford University Press.

Homans, G. C. (1961). Social Behavior: Its elementary forms. New York, NY: Harcourt Brace \& Work.

Hursh, S. R., \& Roma, P. G. (2013). Behavioral economics and empirical public policy. Journal of the Experimental Analysis of Behavior, 99, 98-124.

Kantor, J. R. (1929). An outline of social psychology. Chicago, IL: Follet Publishing Company.

Kantor, J. R. (1936). An objective psychology of grammar. Bloomington, IN: Indiana University Press. doi:10.1037/13513-000

Kantor, J. R. (1982). Cultural psychology. Chicago, IL: Principia Press.

Kropotkin, P. (1902/2008). Mutual Aid. A factor of evolution. Charleston, SC: Forgotten Books.

Lindsley, O. (1966). Experimental analysis of cooperation and competition. En T. Verhave (Ed.), The experimental analysis of behavior (pp. 470-501). New York, NY: Appleton Century Crofts.

Okouchi, H. (2012). Human responding with mutual reinforcement: Baseline and effects of an intervention. Japanese Psychological Research, 54(2), 202-209. doi:10.1111/ j.1468-5884.2011.00500.x

Osborne, M. (2004). An introduction to game theory. Oxford, England: Oxford University Press. 
Pennisi, E. (2005). How did cooperative behavior evolve? Science, 309(93). doi:10.1126/ science.309.5731.93

Pulido, L., Rangel, N., \& Ortiz, G. (2013). El papel del intercambio verbal en la solución de tareas en niños de primaria. Acta Comportamentalia, 21(1), 36-52.

Ribes, E. (2001). Functional dimensions of social behavior: Theoretical considerations and some preliminary data. Revista Mexicana de Análisis de la Conducta, 27, 285-306.

Ribes, E. (2010). Social interactions: Conceptual reflections and an experimental approach. En R. Schwarzer, \& P. A. Frensch (Eds.), Personality, human development and culture: International perspectives on psychological science (pp. 275-288). New York, NY: Psychology Press.

Ribes, E., \& López, F. (1985). Teoría de la conducta un análisis de campo y paramétrico. México, D.F.: Trillas.

Ribes, E., \& Rangel, N. (2002). Choice between individual and shared contingencies in children and adults. European Journal of Behavior Analysis, 3, 61-73.

Ribes, E., Rangel, N., Carbajal, G., \& Peña, E. (2003). Choice between individual and shared social contingencies in children: An experimental replication in a natural setting. European Journal of Behavior Analysis, 4, 105-114.

Ribes, E., Rangel, N., Casillas, J., Álvarez, A., Gudiño, M., Zaragoza, A., \& Hernández, H. (2003). Inequidad y asimetría de las consecuencias en la elección entre contingencias individuales y sociales. Revista Mexicana de Análisis de la Conducta, 29, 385-401. doi:10.5514/rmac.v29.i2.25401

Ribes, E., Rangel, N., Juárez, A., Contreras, S., Abreu, A., Gudiño, M., \& Casillas, J. (2003). Respuestas "sociales" forzadas y cambio de preferencias entre contingencias individuales y sociales en niños y adultos. Acta Comportamentalia, 11, 197-234.

Ribes, E., Rangel, N., \& López, F. (2008). Análisis teórico de las dimensiones funcionales del comportamiento social. Revista Mexicana de Psicología, 25(1) , 45-57.

Ribes, E., Rangel, N., Magaña, C., López, A., \& Zaragoza, A. (2005). Efecto del intercambio diferencial equitativo e inequitativo en la elección de contingencias sociales de altruismo parcial. Acta Comportamentalia, 13, 159-179.

Ribes, E., Rangel, N., Ramírez, E. Valdez, U., Romero, C., \& Jiménez, C. (2008). Verbal and-nonverbal induction of reciprocity in a partial-altruism social interaction. European Journal of Behavior Analysis, 9, 53-72.

Ribes, E., Rangel, N., Zaragoza, A., Magaña, C., Hernández, H., Ramírez, E., \& Valdez, U. (2006). Effects of differential and shared consequences on choice between individual and social contingencies. European Journal of Behavior Analysis, 7, 41-56.

Rostek, M. (2010). Quantile maximization in decision theory. Review of Economic Studies, 77, 339-371, available via: 10.1111/j.1467937X.2009.00564.x

Schmitt, D. (1998). Social Behavior. En K. Lattal y $M$. Perone (Eds.), Handbook of research methods in human operant Behavior (pp. 475503). New York, NY: Plenum Press.

Schuster, R., \& Perelberg, A. (2004). Why cooperate? An economic perspective is not enough. Behavioural Processes, 66, 261-277. doi:10.1016/S0376-6357(04)00057-9

Shubik, M. (1982). Game theory in the social sciences. Cambridge, MA: The MIT Press.

Skinner, B. F. (1953). Science and human behavior. New York, NY: Macmillan.

Skinner, B. F. (1962). Two "synthetic social relations". Journal of the Experimental Analysis of Behavior, 5, 531-533. doi:10.1901/ jeab.1962.5-531

Stephens, D. W., \& Anderson, J. P. (1997). Reply to Roberts: cooperation is an outcome, not a mechanism. Animal Behavior, 53(6), 13631364. doi:10.1006/anbe.1996.0379

Weiner, H. (1977). An operant analysis of human altruistic responding. Journal of the Experimental Analysis of Behavior, 27, 515528. doi:10.1901/jeab.1977.27-515

Weingarten, K., \& Mechner, F. (1966). The contingency as an independent variable of social interaction. En T. Verhave (Ed.), The Experimental Analysis of behavior (pp. 449459). New York, NY: Appleton-Century-Crofts.

Weiss, A. P. (1926). A set of postulates for social psychology. Journal of Abnormal and Social 
Psychology, 21, 203-211. doi:10.1037/ h0074344

Wittgenstein, L. (1953). Philosophical Investigations. (G.E.M. Anscombe, Trad.). Oxford, England: Basil Blackwell.

Wittgenstein, L. (1958). The blue and brown books. Oxford, England: Basil Blackwell.

Yi, R., \& Rachlin, H. (2004). Contingencies of reinforcement in a five-person prisoner's dilemma. Journal of the Experimental Analysis of Behavior, 82(2), 161-176. doi:10.1901/ jeab.2004.82-161

Auto-referencias de autores: 9

Auto-referencias de revista JBHSI: 0 